\title{
La Société des agrégées, entre féminisme et esprit de catégorie (1920-1948)
}

The Société des agrégées, between Feminism and esprit de corps (1920-1948) Die „Société des agrégées“ zwischen Feminismus und korporativem Denken (1920-1948)

La Société des agrégées, entre feminismo y espíritu de categoría (1920-1948)

\section{Yves Verneuil}

\section{(2) OpenEdition}

\section{Journals}

Édition électronique

URL : https://journals.openedition.org/histoire-education/1426

DOI : $10.4000 /$ histoire-education. 1426

ISSN : 2102-5452

\section{Éditeur}

ENS Éditions

\section{Édition imprimée}

Date de publication : 1 septembre 2007

Pagination : 195-224

ISBN : 978-2-7342-1101-3

ISSN : 0221-6280

\section{Référence électronique}

Yves Verneuil, «La Société des agrégées, entre féminisme et esprit de catégorie (1920-1948) », Histoire de l'éducation [En ligne], 115-116 | 2007, mis en ligne le 01 janvier 2012, consulté le 20 mai 2021. URL: http://journals.openedition.org/histoire-education/1426 ; DOI : https://doi.org/10.4000/histoireeducation. 1426 


\title{
LA SOCIÉTÉ DES AGRÉGÉES, entre féminisme et esprit de catégorie (1920-1948)
}

\author{
par Yves VERNEUIL
}

Institué en 1880 par la loi Camille Sée, l'enseignement secondaire féminin est doté d'un personnel exclusivement féminin, afin de ne pas exposer les établissements de jeunes filles aux critiques dont les conservateurs avaient accablé les cours secondaires établis par Victor Duruy à la fin du Second Empire. Des certificats d'aptitude à l'enseignement secondaire des jeunes filles sont créés en 1882, des agrégations féminines en 1883. Même s'il mécontente les agrégés, qui l'estiment indu, car ne reposant pas sur l'exigence de la licence, le titre des agrégées doit assurer la réputation du nouvel enseignement. Dans les lycées de jeunes filles, les agrégées sont les seuls professeurs titulaires, comme le sont les agrégés dans les établissements masculins. Les certifiées sont affectées indifféremment en collège ou en lycée, avec même traitement. Cette mesure est censée favoriser la stabilité des personnels féminins, car, dans l'enseignement secondaire masculin, les licenciés cherchent à passer des fonctions de professeur de collège à celles de chargé de cours de lycée, afin de bénéficier d'une promotion financière. Aussi bien, le législateur n'a pas voulu seulement distinguer les enseignements masculin et féminin: il a également voulu éviter aux établissements féminins les défauts de leurs homologues masculins, du point de vue des personnels comme du point de vue de la pédagogie. Au début du XX siècle s'amorce pourtant une évolution pédagogique qui va à rebours des intentions de départ: sous la pression de jeunes filles de plus en plus avides de passer le baccalauréat, l'enseignement secondaire féminin se rapproche de son homologue masculin.

Retraçant cette évolution, qui aboutit à l'assimilation des deux enseignements en 1924, Françoise Mayeur mentionne la contribution 
qu'y a apportée la Société des agrégées (1). Cette indication invite à une nouvelle thématique, externe à l'enseignement, mais étroitement liée à lui, celle des organisations enseignantes féminines, et donc à la principale d'entre elles, la Société des agrégées (2). Alors que les associations enseignantes sont le plus souvent étudiées sous l'angle de l'idéologie et de la politique, c'est l'occasion de s'intéresser à une organisation catégorielle, à son fonctionnement comme à ses revendications. Cette étude est possible grâce au bulletin corporatif, Les agrégées, qu'a édité la Société, trace importante qui doit être complétée par la lecture des bulletins corporatifs d'autres organisations et par celle de journaux universitaires, qu'ils soient spécialisés, comme L'enseignement secondaire des jeunes filles, ou généralistes, comme la Revue universitaire (qui comporte cependant, depuis 1909, une rubrique spéciale concernant l'enseignement secondaire féminin, tenue par la sévrienne Jeanne Crouzet-Benaben).

Réunissant les titulaires féminines du concours de l'agrégation, la Société des agrégées est fondée sur un esprit de catégorie qui peut paraître peu propice à l'union du personnel féminin. Loin d'être le simple reflet de la division par sexe de l'enseignement secondaire, l'apparition d'une association corporative féminine n'allait en effet pas de soi, si l'on considère que les femmes professeurs avaient adhéré collectivement, en 1905, à la Fédération nationale des professeurs de lycée et du personnel de l'enseignement secondaire féminin, dite $\mathrm{A} 3$, et que leur lutte contre la domination masculine aurait pu tout aussi bien déboucher sur la création d'une fédération féminine indépendante. D'un autre côté, cependant, dans la mesure où elle s'est fondée en réaction à l'hégémonie de la Société masculine sur l'A3, la Société des agrégées a eu intérêt à insister sur la solidarité féminine, pour contrer la domination des agrégés. Quelle attitude allait-elle donc privilégier? Serait-elle prioritairement un mouvement féministe luttant en faveur de l'égalité des droits entre hommes et femmes, ou bien une organisation visant à maintenir la prééminence des agrégées dans l'enseignement secondaire féminin? Son ambi-

(1) Françoise Mayeur, L'enseignement secondaire des jeunes filles sous la Troisième République, Paris, Presses de la FNSP, 1977.

(2) Cf. Yves Verneuil, La Société des agrégés, de sa fondation à nos jours, doctorat d'histoire, sous la direction de Françoise Mayeur, université Paris IV, 2001. $C f$. aussi Martine Walker, « Un aspect des luttes féminines dans l'enseignement secondaire: la Société des agrégées (1920-1948) », Pénélope. Pour une histoire des femmes, $\mathrm{n}^{\circ} 2$, printemps 1980, pp. 61-63. 
guité constitutive doit être étudiée sur le temps long, afin de comprendre l'évolution qu'elle a connue entre son apparition, en 1920, et sa fusion avec la Société des agrégés en 1948.

\section{LA FONDATION (1920)}

Ce n'est pas la question du rapprochement des enseignements secondaires masculin et féminin qui est à l'origine de la Société des agrégées. Sur ce point, en effet, les femmes professeurs sont divisées. Depuis 1909, la future fondatrice de la Société des agrégées, Claire Suran-Mabire, professeur à Marseille, est convaincue de la nécessité de l'assimilation entre les deux enseignements, d'un point de vue pédagogique comme d'un point de vue catégoriel; mais d'autres femmes, telles Hélène Guénot, professeur au lycée Racine (1), et même Élisabeth Butiaux, la future première présidente de la Société des agrégées, tiennent à conserver la spécificité de l'enseignement secondaire féminin.

La question du rapprochement des enseignements secondaires masculin et féminin a cependant mis en évidence l'infériorité statutaire des femmes; la comparaison des deux enseignements a rendu plus évident le lien entre la spécialisation limitée des concours féminins (2) et les conditions de travail moins favorables des femmes professeurs (traitements inférieurs, services supérieurs). Craignant un déclassement si des femmes exercent le même métier qu'eux, des professeurs hommes ne manquent pas de rappeler que leurs collègues féminines ne sont pas assez qualifiées pour préparer au baccalauréat: les chargés de cours déclarent régulièrement que le certificat d'aptitude à l'enseignement secondaire des jeunes filles, dénué de spécialisation, n'est qu'un « super brevet supérieur » (injure suprême). Même les agrégées n'ont pas la formation classique requise: leur niveau, murmurent leurs collègues masculins, est douteux, leur spécialisation

(1) Hélène Guénot, « Contre l'identification des programmes masculins et des programmes féminins », La Revue universitaire, T. II, 1916, pp. 118-126.

(2) Les agrégations féminines comprennent quatre sections (lettres, histoire, mathématiques, sciences physiques et naturelles), alors que les agrégations masculines comportent huit ordres (grammaire, lettres, philosophie, histoire, mathématiques, sciences physiques, sciences naturelles, langues vivantes). Le certificat d'aptitude à l'enseignement secondaire féminin comprend seulement deux sections (lettres et langues). Cf. André Chervel, Histoire de l'agrégation. Contribution à l'histoire de la culture scolaire, Paris, INRP/Kimé, 1993. 
insuffisante; l'agrégation féminine de lettres ne comporte ni latin, ni grec; sauf à le dévaluer, les femmes ne sauraient donc préparer au baccalauréat. Chez les plus radicales des femmes professeurs se renforce, de ce fait, l'idée qu'il faut assimiler les concours masculins et féminins. Selon Claire Mabire, cette assimilation permettrait de hisser les agrégées au niveau de rémunération des agrégés (1). Ce serait aussi le moyen de leur procurer la spécialisation qui, dans l'enseignement masculin, va de pair avec un service déterminé (2). Ce projet met toutefois en porte-à-faux les certifiées, qui s'estiment issues d'épreuves autrement plus sélectives que la licence, mais qui n'en sont pas moins considérées comme inférieures par les professeurs masculins licenciés. Quelle serait leur place dans une configuration assimilationniste, qui exigerait une spécialisation?

Cependant, l'émergence catégorielle des agrégées est ralentie par le sentiment de solidarité féminine. En 1905, au moment où se crée la Fédération nationale des professeurs de lycée, le personnel de l'enseignement secondaire féminin, du fait de son isolement dans la société, est plus sensible à ce qui fait l'unité de sa condition qu'à sa diversité et adhère collectivement à la Fédération (tandis que les professeurs masculins de collège et les répétiteurs ont fondé des fédérations séparées) (3). Le désir des femmes professeurs de rester unies se marque lors du dépôt des vœux devant la Commission extraparlementaire sur la revalorisation des traitements, en 1907: alors que chaque catégorie masculine présente ses revendications en combattant celles des autres, les femmes commencent par solliciter un délai, afin de se

(1) Jusqu'en 1910, le traitement maximum d'un chargé de cours des départements (4800 francs) est supérieur à celui de l'agrégée des départements (4200 francs) et même à celui de l'agrégée du cadre de Paris (4700 francs). Tout se passe comme si la licence valait plus que l'agrégation féminine. Le traitement de l'agrégé masculin peut atteindre 7500 francs à Paris, 5200 francs (non compris l'indemnité d'agrégation, de 500 francs) dans les départements.

(2) Même après la réforme de 1894, les agrégations féminines restent peu spécialisées, si bien que l'administration continue à pouvoir demander à une agrégée de lettres d'assurer une partie de son service en histoire. De là un service plus fatigant et un nombre restreint d'heures supplémentaires (lorsqu'il reste des heures, on les attribue au professeur d'une autre matière dont le service est incomplet).

(3) L'adhésion groupée du personnel de l'enseignement secondaire féminin s'explique plus par ce sentiment d'unité que par l'exemple du « féminisme universitaire », à ses débuts. Celui-ci, au reste, concerne surtout le personnel de l'enseignement primaire: $c f$. Jacques Girault, Instituteurs, professeurs, une culture syndicale dans la société française (fin XIXe-XXe siècle), Paris, Publications de la Sorbonne, 1996, p. 141. 
mettre d'accord pour établir un tableau d'ensemble (1). Par ailleurs, en 1913, les agrégées soutiennent les chargés de cours des lycées dans leur revendication d'assimilation financière avec les agrégés, au nom du principe « à travail égal, salaire égal ». Cette alliance avec les chargés de cours est considérée comme une " trahison » par les agrégés. Quand, en 1914, ceux-ci fondent une société particulière afin de faire pression sur la Fédération nationale, ils en excluent donc les femmes: "Les agrégées de l'enseignement féminin ne sont pas admises », précise le commentaire des statuts fondateurs de la Société des agrégés de l'enseignement secondaire (2).

Des indices témoignent néanmoins qu'une conscience de grade plus aiguë se forme chez les agrégées. La génération de Claire SuranMabire a souffert, en début de carrière, du manque d'emplois consécutif à la stagnation des effectifs des établissements féminins. Cette situation a conduit à affecter des agrégées dans des collèges, avec un traitement qui n'était pas celui de leur grade, mais celui de leur fonction provisoire. L'initiatrice de la Société des agrégées a elle-même ressenti péniblement cette affectation sans rapport avec ce qu'elle estimait être sa compétence. Aussi bien, quand elle dénonce les conditions de travail des femmes professeurs, qui sont sans rapport, selon elle, avec leur niveau d'études, c'est le cas des agrégées qu'elle a en tête (3).

Après la guerre, l'inflation attise les passions catégorielles au sein de la Fédération nationale des professeurs de lycée, car chaque catégorie exige un relèvement de son traitement sans se préoccuper de celui des autres catégories (4). Les hommes se soucient tellement peu des revendications de leurs collègues féminines qu'ils quittent en général les réunions quand il est question de l'enseignement secondaire féminin. Les femmes peuvent donc se sentir négligées. Il n'est toutefois pas question de fonder une fédération féminine séparée. En revanche, Claire Suran-Mabire, vice-présidente de l'A3, estime que les agrégées doivent prendre modèle sur les hommes en fondant une

(1) $\mathrm{AN} \mathrm{F}^{17} 12748$

(2) L'Agrégation, $\mathrm{n}^{\circ}$ 1, mai 1914, p. 9.

(3) Claire Suran-Mabire, «L'enseignement secondaire féminin et la crise du recrutement », Revue universitaire, T. 2, 1914, pp. 37-49.

(4) Sur la question des traitements, $c f$. Yves Verneuil, Les agrégés. Histoire d'une exception française, Paris, Belin, 2005, pp. 159-162. 
association catégorielle qui veillera à ce que la Fédération nationale prenne en compte leurs revendications en matière de traitement et de service et publie un appel en ce sens dans Le Journal des lycées (1). Elle pense d'ailleurs que les certifiées et les autres catégories de l'enseignement secondaire féminin devraient créer, elles aussi, leurs associations particulières. On l'accuse de briser l'unité des femmes professeurs au sein de la Fédération nationale (2). Elle réplique:

Les agrégées, les certifiées, les licenciées, les diplômées, les bachelières et les brevetées peuvent enseigner les mêmes matières dans des établissements divers. Ce n'est pas une raison pour que leurs « cahiers » de revendications ne soient pas distincts. L'union n'est pas dans la confusion [...]. La répartition du travail n'a rien de commun avec la division des esprits (3).

Pour elle, les femmes doivent constituer des sociétés de catégorie qui seront autant d'armées alliées en perpétuelle liaison au sein de la Fédération. Le risque était pourtant que ces armées finissent par se déchirer. L'exemple masculin n'était pas très encourageant. En tout état de cause, l'ambiguïté originelle de la Société, entre esprit de catégorie et union féminine, se trouve exprimée dans cette réponse.

Cependant, Claire Suran-Mabire a agi de son propre mouvement: les agrégées parisiennes ont été prises de court (4). Une entente survient néanmoins, qui permet la tenue de la réunion constitutive de la Société des agrégées au lycée Fénelon, le 17 juillet 1920 (5). Claire Suran-Mabire en devient vice-présidente, mais la présidence lui échappe au profit d'Élisabeth Butiaux. Ce choix n'est pas innocent. Élisabeth Butiaux a exercé, au lycée Fénelon, toutes les fonctions d'enseignement à l'intérieur de l'enseignement secondaire féminin: maîtresse primaire pourvue du brevet supérieur, professeur certifiée, puis professeur agrégée. Au moment où les autres catégories s'inquiètent du manque de solidarité que semble signifier la fondation

(1) Le Journal des lycées, $\mathrm{n}^{\circ} 23$, mars 1920, pp. 1-2.

(2) Le Journal des lycées, $\mathrm{n}^{\circ} 25$, mai 1920, p. 1.

(3) Claire Suran-Mabire, "Société de catégorie et union fédérale », Le Journal des lycées, $\mathrm{n}^{\circ} 27$, juillet 1920, pp. 1-2.

(4) Le corps des agrégées, comme celui des agrégés, comprend deux cadres, le cadre des départements et le cadre de Paris, avec deux échelles différentes de traitement. L'accès au cadre de Paris se fait au choix, sur proposition de l'inspection générale. Les Parisiennes ont donc l'impression d'appartenir à une élite.

(5) Les Agrégées, $\mathrm{n}^{\circ}$ 1, octobre 1920, p. 2. 
d'une Société des agrégées, cette figure peut rassurer sur la volonté de maintenir un lien avec elles. Son hostilité à l'identification des enseignements masculin et féminin rassure, par ailleurs, les agrégées traditionalistes: on peut réclamer la parité des traitements tout en sauvegardant la spécificité de l'enseignement féminin.

Les statuts de la Société féminine sont copiés sur ceux de la Société masculine. L'article 2 précise toutefois: «La Société des agrégées désire collaborer avec toutes les autres catégories de l'enseignement secondaire, et particulièrement de l'enseignement féminin, et se joindre à elles dans leurs justes revendications ». La fierté catégorielle n'exclut donc pas le sentiment de solidarité féminine. Les agrégées se présentent comme les porte-parole naturels de l'enseignement secondaire féminin, tout en prétendant rester unies avec les autres professeurs.

\section{LES LUTTES SUCCESSIVES (1920-1933)}

Jusqu'en 1933, l'histoire de la Société des agrégées est une suite de conquêtes dans le sens de l'égalité entre les hommes et les femmes. Cette politique offensive - toujours mesurée dans le ton comme dans la forme - distingue la Société des agrégées de son homologue masculine, préoccupée de la défense des positions des agrégés contre les menaces assimilationnistes des chargés de cours. La Société des agrégées a pris, de ce fait, une tournure progressiste. Cet état d'esprit est renforcé, chez les agrégées, par la volonté de promouvoir le caractère « moderne » de leur enseignement, par une plus grande ouverture aux questions pédagogiques et par le souvenir de la solidarité catégorielle des professeurs de l'enseignement secondaire féminin.

Il n'est d'ailleurs pas question de collaboration avec la Société masculine. Au vrai, celle-ci s'oppose régulièrement aux vœux de son homologue féminine, sous prétexte que les agrégations féminines n'auraient pas la même valeur. C'est pourquoi la Société des agrégées, qui est destinée à regrouper non seulement les titulaires des agrégations féminines, mais aussi les titulaires des agrégations masculines (celles qu'on appelle les « agrégées masculines ») (1), est très

(1) La Société regroupe effectivement $80 \%$ des agrégées et passe de 194 adhérentes en 1921 à 1111 en 1939. 
défavorable à ce que ces dernières puissent adhérer à la Société des agrégés (1). $\mathrm{M}^{\mathrm{Hle}}$ Bois, professeur de philosophie au lycée du Mans, qui aurait voulu adhérer à la Société masculine (2), récuse la doctrine de la solidarité féminine, qui aboutit, selon elle, à la « guerre des sexes ». Mais la Société des agrégées persiste à penser que les agrégées doivent conserver leur propre association.

Cette position donne à la Société des agrégées une allure féministe qui est vivement dénoncée par le camp antiféministe de la Société des agrégés, emmené par Jean Maillan (3). Et pourtant, en 1921, la Société a refusé l'adhésion au Conseil national des femmes (CNF), qu'elle trouve trop politique:

Le Conseil national des femmes et la Ligue pour les droits de la femme nous ayant, à plusieurs reprises, laissé entendre que notre cause gagnerait à être défendue par leurs groupes, $\mathrm{M}^{\text {elle }}$ Véroux a dit à $\mathrm{M}^{\text {Hle }}$ Desprez [rapporteur de la section «Éducation » au CNF] ce qu'elle croit être notre pensée à toutes: nous serons toujours reconnaissantes aux sociétés féministes de l'appui que leur action peut apporter à la nôtre, nous serons heureuses de nous tenir en rapport avec elles, mais il est de meilleure guerre qu'elles et nous suivions des voies parallèles et non pas conjuguées (4).

C'est donc en s'appuyant sur la Fédération nationale des professeurs de lycée (ensuite devenu Syndicat national) (5), dont elle est d'ailleurs officiellement une composante, que la Société parvient à arracher aux pouvoirs publics ses revendications, les unes après les autres.

(1) Au demeurant, en 1928 comme en 1930, la Société masculine refuse d'accueillir les « agrégées masculines »: cf. L'Agrégation, nº 99, mai 1930, pp. 395399.

(2) L'Agrégation, $\mathrm{n}^{\circ} 87$, novembre 1929, pp. 80-81.

(3) C'est Jean Maillan qui est le plus hostile à l'idée d'ouvrir la Société des agrégés aux femmes titulaires d'agrégations masculines. En 1928, il met ainsi en garde ses collègues: "La solidarité de sexe jouant une fois de plus [...] contre nous, $[\ldots]$ les nouvelles venues, c'est fatal, feront la courte échelle à leurs aînées. Ce sera la ruée à nos portes qui, entr'ouvertes imprudemment par nous aujourd'hui, livreront passage demain à l'armée des femmes tout entière. N'oublions pas le Cheval de Troie » (L'Agrégation, $\mathrm{n}^{\circ}$ 5, mars 1928, pp. 24-29). Sur cet antiféminisme dominé par la peur, cf. Christine Bard, « Pour une histoire des antiféministes », in Christine Bard, Un siècle d'antiféminisme, Paris, Fayard, 1999, p. 26.

(4) Les Agrégées, $\mathrm{n}^{\circ}$ 4, novembre 1921, p. 4.

(5) En 1925, la Fédération nationale des professeurs de lycée et du personnel de l'enseignement secondaire féminin (A3) se transforme en syndicat (S3). 


\section{L'identification des enseignements}

Ce n'est que progressivement que la Société s'est ralliée à l'idée d'une identification sans condition de l'enseignement secondaire féminin à son homologue masculin. Pour Élisabeth Butiaux, on peut obtenir l'égalité des traitements sans réaliser l'unification des enseignements et des concours: on peut spécialiser les chaires des agrégées sans que cela implique l'alignement des programmes féminins sur les programmes masculins. D'ailleurs, le congrès de l'A3, en 1920, a admis que l'unification des concours ne devait pas être un préalable à l'égalité des traitements. Rapidement, toutefois, une majorité se dégage au sein du comité de la Société sur l'idée qu'il faut rechercher l'identité des deux enseignements secondaires. Une égale dignité semble en effet difficile à atteindre si les enseignements sont différents, car les misogynes auront toujours beau jeu de prétendre que l'enseignement féminin est inférieur. C'est notamment l'argumentation de Gabrielle Véroux et de Catherine Schulhof. Fières de leurs propres études, persuadées de l'égalité intellectuelle des femmes et des hommes, ces agrégées veulent que les jeunes filles puissent accéder normalement aux études supérieures, ce qui suppose qu'elles passent le baccalauréat et, par conséquent, l'identité des études secondaires des garçons et des filles. La circulaire du 23 octobre 1922 (1), qui autorise les jeunes filles passant la deuxième partie du baccalauréat à suivre les cours des classes de philosophie ou de mathématiques dans les lycées de garçons si aucune classe n'est créée à cet effet dans les lycées de jeunes filles, est au reste une menace pour l'enseignement féminin.

La question est de savoir ce que doit être cet enseignement secondaire destiné aussi bien aux jeunes filles qu'aux jeunes gens. Pour nombre de femmes professeurs, l'enseignement secondaire féminin ne se borne pas à instruire, il propose aussi une éducation; c'est un véritable enseignement de culture, alors que l'enseignement secondaire masculin ne connaît que le bachotage. Pourquoi vouloir à tout prix l'identité des enseignements masculin et féminin, persistent donc à demander les traditionalistes, très présentes notamment au sein de la revue L'enseignement secondaire des jeunes filles? L'hésitation de certaines agrégées est accrue par les projets ministériels concernant la

(1) Bulletin administratif du ministère de l'Instruction publique, $\mathrm{n}^{\circ} 2500$, 15 novembre 1922, p. 481. 
réforme de l'enseignement secondaire masculin: Léon Bérard voudrait revenir sur la réforme de 1902 et supprimer la section moderne. Or les traditionalistes considèrent que l'enseignement littéraire moderne fait partie des acquis dont il serait dommage que l'enseignement féminin soit privé. On peut en outre trouver curieux que le sort des agrégées soit suspendu à celui de l'enseignement masculin: situation en effet paradoxale de la part de «féministes ». C'est ce que fait observer Hélène Guénot, qui refuse de se rallier à la revendication d'assimilation de l'enseignement féminin à celui des garçons, devenue majoritaire à la Société des agrégées dès 1921. H. Guénot condamne un «féminisme masculin » qui s'appliquerait à copier les hommes, ce à quoi aboutit la doctrine de l'assimilation. Selon elle, Camille Sée avait été plus courageux en créant un enseignement féminin spécifique, conforme à un féminisme vraiment « féminin ». Face à ces critiques, la Société est amenée, tout en défendant la doctrine de l'assimilation, à rassurer aussi bien les agrégées que les agrégés. En 1921, Gabrielle Véroux écrit ainsi aux agrégés membres du Conseil supérieur de l'Instruction publique (CSIP):

Nous demandons au Conseil supérieur de faire confiance aux professeurs femmes sur ce point: quels que soient les programmes, les sanctions de l'enseignement secondaire réorganisé, elles formeront des épouses, des mères et même des ménagères, des femmes enfin, du moins autant que par le passé (1).

Ainsi la Société s'inscrit-elle malgré tout, du moins dans les années 1920, dans le courant du féminisme majoritaire entre les deux guerres, qui cherche à garder à la femme ses caractères dits féminins (2). La revendication d'assimilation des deux enseignements n'en est pas moins acquise. Le 21 décembre 1921, la délégation des agrégées devant le CSIP déclare qu'elle demandera l'unification des études secondaires, même si la réforme de l'enseignement masculin est « conçue de manière à ne satisfaire à aucun des vœux exprimés dans nos réponses » (3), c'est-à-dire même en cas de suppression de la filière moderne.

Il n'empêche que la perspective d'une réforme de la filière classique est redoutée même par les assimilationnistes. Si le latin est

(1) Les Agrégées, $\mathrm{n}^{\circ}$ 4, novembre-décembre 1921, p. 7.

(2) Michèle Riot-Sarcey, Histoire du féminisme, Paris, La Découverte, pp. 76-77.

(3) Les Agrégées, n 5, mars 1922, p. 8. 
obligatoire en sixième, il sera en effet encore plus difficile de faire fusionner les enseignements secondaires féminin et masculin, dans la mesure où l'on représentera que les jeunes filles ne sont pas aptes à faire de telles études et que les actuelles agrégées de lettres ne sont pas qualifiées. C'est pourquoi, en décembre 1921, par référendum interne, la Société des agrégées se prononce contre le projet de suppression de la section moderne de l'enseignement secondaire. En novembre 1922, elle fait de nouveau savoir son hostilité à l'obligation du latin et du grec dans tout le secondaire et va jusqu'à prôner la création d'une agrégation de lettres modernes. Le 4 avril 1923, l'Assemblée générale condamne fermement le projet ministériel.

En janvier, le CSIP, malgré son hésitation, en a fait autant. Cependant, en dépit des critiques de la presse de gauche, qui condamne les implications sociales de la réforme, et de la rhétorique d'Édouard Herriot, qui compare les méthodes du ministre à celles du duc de Broglie sous le gouvernement de l'Ordre moral (1), Léon Bérard supprime, par décret du 3 mai, la section moderne du premier cycle. L'article 10 du décret annonce que seront pris ultérieurement des décrets pour adapter le nouveau plan d'études à l'enseignement secondaire des jeunes filles. Mais, comme le souligne l'agrégé de philosophie Georges Beaulavon devant le Conseil supérieur, la réforme complique l'unification des enseignements secondaires féminin et masculin (2).

Le ministre n'en décide pas moins l'alignement de l'enseignement secondaire féminin sur son homologue masculin par le décret du 25 mars 1924. Comme elle l'avait annoncé, en dépit de son hostilité à la réforme de l'enseignement masculin, la Société des agrégées accepte cette mesure. Cependant, après la victoire électorale du Cartel des Gauches, elle applaudit au rétablissement de la section moderne.

Dans la lutte entre « classiques » et « modernes » qui divise les professeurs masculins de l'enseignement secondaire, la Société des agrégées s'est donc inscrite dans le clan des « modernes ». Dans la

(1) John E. Talbott, The Politics of Educational Reform in France, 1918-1940, Princeton, Princeton University Press, 1969, pp. 82 et 84.

(2) Revue universitaire, T. 2, 1924, pp. 129-130, cité par Nicole Hulin, Les femmes et l'enseignement scientifique, Paris, PUF, 2002, p. 82. 
mesure où l'Action française et la droite conservatrice, depuis la réforme de 1902, ont assimilé l'identité française à la défense des humanités classiques, la position de la Société des agrégées contribue à la ranger dans le camp progressiste, même si, en l'occurrence, c'est pour préserver la tradition de l'enseignement secondaire féminin et si bien des « classiques », parmi les agrégés, sont en vérité des hommes de gauche: conservatisme pédagogique et conservatisme politique ne vont pas de pair. La prise de position de la Société des agrégées en faveur de l'enseignement moderne la différencie en tout cas de son homologue masculine, qui a préféré ne pas prendre position, ses membres étant trop partagés.

Même si elles sont divisées sur la destinée sociale des jeunes filles, toutes ne partageant pas l'idée que les lycéennes doivent entreprendre des études supérieures pour se préparer à une vie professionnelle, les agrégées ont majoritairement œuvré au processus d'assimilation des enseignements secondaires, qui permet aux jeunes filles de prouver qu'elles ont une intelligence égale à celle des jeunes gens. Ce combat, toutefois, n'a pas été exempt d'arrière-pensées: le féminisme se teinte d'esprit catégoriel. Preuve en est que, contrairement à celle des agrégées, la Société des certifiées s'est prononcée pour le maintien de la section préparant au diplôme de fin d'études secondaires, spécifique à l'enseignement féminin: ce n'est pas un hasard. Les certifiées ont intérêt à protéger la spécificité de l'enseignement féminin, puisque la spécialisation des chaires, et donc des concours, qu'implique l'identité des enseignements masculin et féminin paraît plus facile à réaliser pour les agrégations féminines, qui ont connu un début de spécialisation en 1894, que pour les certificats d'aptitude. De fait, les agrégées revendiquent l'unification des concours masculins et féminins, sans toutefois qu'il soit précisé si cela implique la mixité des concours ou simplement la spécialisation des agrégations féminines sur le modèle masculin.

\section{L'unification des concours de recrutement}

En 1917, dans leurs réponses à la Commission extraparlementaire sur l'enseignement féminin, les femmes s'étaient montrées partagées sur la nécessité d'unifier les concours masculins et féminins (1). Une

(1) Françoise Mayeur, op. cit., p. 405. 
fois constituée, la Société des agrégées, en revanche, se prononce d'emblée pour leur unification. L'assimilation des enseignements rend la question pressante si l'on ne veut pas que les hommes enseignent dans les établissements féminins ou que les jeunes filles se rendent massivement dans les établissements masculins. Huit jours avant le décret instaurant l'assimilation des enseignements, Léon Bérard décide, par arrêté du 17 mars 1924, que tous les concours et certificats masculins seront désormais ouverts aux femmes. Pour la Société, cette mesure est insuffisante: elle maintient des agrégations féminines, dont les titulaires ne seront peut-être jamais reconnues à égalité.

En 1926, le ministère accepte le principe de l'unification des concours, dont l'échéance est prévue pour 1938. Les agrégations féminines sont progressivement spécialisées. La mixité des agrégations n'est toutefois pas réalisée; les agrégations féminines sont maintenues. Contrairement à ses intentions de départ, la Société des agrégées paraît s'en contenter. Il est vrai que la mixité des concours aurait pu déboucher sur l'interchangeabilité des personnels masculins et féminins, et donc priver les femmes de certaines chaires des lycées de jeunes filles, sans qu'elles soient sûres d'en conquérir dans les établissements masculins. La mixité des concours aurait facilité la comparaison intellectuelle avec les hommes, mais cet objectif risquait d'entrer en opposition avec la défense des postes. La Société des agrégées a préféré mettre en sourdine ses revendications féministes plutôt que de heurter l'intérêt catégoriel de ses adhérentes. De toute façon, la question était certainement devenue moins pressante depuis qu'en 1927, l'égalité des traitements avait été réalisée.

\section{L'égalité des traitements et des maxima de service}

La question des traitements est la question-clé. C'est elle qui a motivé la création de la Société des agrégées. Celle-ci demande l'égalité des traitements, même si l'unification des concours ne se réalise pas, ou du moins sans attendre qu'elle se réalise. Cette exigence complique évidemment les débats et facilite la tâche des agrégés hostiles à l'égalité financière. Ceux-ci ont beau jeu de représenter qu'accorder l'égalité financière à des femmes dont le titre est d'un niveau (selon eux) moins élevé que le titre masculin correspondant serait encourager la revendication des chargés de cours à l'assimilation financière, voire que la dévalorisation des titres conduit droit à la primarisation 
de l'enseignement secondaire. Ce jugement négatif porté sur les agrégations féminines explique que la Société féminine fasse tout pour convaincre les « agrégées masculines » de ne pas chercher à adhérer à la Société masculine. Toutes les agrégées doivent être solidaires, souligne la Société des agrégées, car ce qui est en jeu n'est pas une question de titre, mais de sexe: une agrégée masculine perçoit le traitement d'une agrégée féminine non seulement si elle enseigne dans un lycée féminin, mais également si elle enseigne dans un lycée de garçons, alors qu'elle a les mêmes titres et exerce rigoureusement la même fonction que son collègue masculin.

En 1920, après de tumultueux débats, l'A3 a admis le principe de l'égalité des traitements entre les personnels masculin et féminin de l'enseignement secondaire. Les agrégés hostiles à l'égalité financière n'ont toutefois pas désarmé. Selon Jean Maillan, qui parvient à faire voter par la Société des agrégés une motion contre l'égalité des traitements en l'absence d'égalité des titres, les professeurs hommes favorables à l'égalité des traitements sans condition sont victimes d'une « idéologie, d'un principe métaphysique: l'égalité de l'homme et de la femme ». Pour empêcher les agrégées de parvenir à leurs fins, Jean Maillan met au point une habile stratégie: il propose, avant le congrès de 1922, que les certifiées bénéficient de traitements plus élevés que les licenciés professeurs de collège. Cette proposition représente une évolution par rapport à l'avant-guerre, au cours duquel les professeurs chargés de cours, c'est-à-dire licenciés, s'estimaient titulaires d'un titre indiscutablement supérieur à celui des certifiées (dont l'absence de spécialisation était présentée comme «primaire ») et peut-être même à celui des agrégées. Avec sa proposition, Jean Maillan fait en réalité coup double: il brise la solidarité féminine tout en dévalorisant la licence, ce qui porte atteinte aux revendications assimilationnistes des chargés de cours. La présidente de la Société des agrégées, Emma Flobert, considère la menace comme suffisamment grave pour aller s'assurer du soutien de la présidente de la Société des certifiées, Melle Saladin. Celle-ci la rassure: n'est-ce pas grâce à la Société des agrégées que les certifiées ont obtenu, l'année précédente, l'égalité de traitement avec les licenciés des collèges? L'assemblée générale de la Société des agrégées se prononce en tout cas contre la possibilité que les certifiées, dont le diplôme a été assimilé à la licence, bénéficient d'un traitement supérieur à celui des professeurs licenciés de collège; elle veut bien demander une indemnité de certificat pour les certifiées, mais elle tient à l'équivalence entre les traitements et les titres et elle 
exige, en revanche, que les chargées de cours soient autant payées que leurs homologues masculins (1). Cette revendication coïncide avec un point de vue nouveau sur les certifiées, affectées indifféremment en collège ou en lycée: la Société des agrégées demande que celles-ci commencent par enseigner en collège, puis puissent être nommées chargées de cours dans un lycée, par promotion au choix, comme cela se pratique dans l'enseignement masculin. Le traitement des chargées de cours en serait augmenté, mais surtout il deviendrait encore plus apparent que les agrégées sont les seuls professeurs de plein droit dans les lycées, puisque les certifiées ne pourraient accéder directement à ces établissements: seules les meilleures d'entre elles pourraient y être nommées, ce qui validerait l'idée, courante à la Société masculine, mais à cette date pas énoncée explicitement dans la Société féminine, selon laquelle l'agrégation est la preuve du talent pédagogique. Le modèle masculin favorise donc les agrégées; le projet d'assimilation des enseignements secondaires masculin et féminin a des soubassements catégoriels. Ce changement de doctrine de la Société des agrégées au sujet de la place des certifiées coïncide, au reste, avec le remplacement de $\mathrm{M}^{\text {lle }}$ Saladin, hostile à l'unification des enseignements, par Catherine Schulhof, membre du comité de la Société des agrégées, à la vice-présidence de la Fédération nationale des professeurs de lycée.

En 1922, un référendum au sein de cette dernière maintient le principe de l'égalité des traitements sans attendre l'unification des concours, en dépit de l'hostilité de la majorité des agrégés. En 1927, le principe de l'égalité est admis par les pouvoirs publics (2). L'égalité des services est évidemment l'étape suivante à atteindre: sans elle, l'égalité des traitements serait une duperie. Les agrégées ont en effet un service de seize heures hebdomadaires, contre quinze au plus pour les hommes. Elles n'ont pas bénéficié des réductions accordées aux hommes en 1911. Une agrégée de philosophie exerçant dans un lycée parisien a un service plus lourd de cinq heures que son collègue masculin exerçant dans le lycée voisin. Même un agrégé de philosophie enseignant dans un lycée féminin a un service de onze heures.

(1) Contrairement aux pratiques de l'enseignement masculin, l'enseignement secondaire féminin rémunère de la même manière professeurs de collège et chargées de cours de lycée.

(2) L'obtention de l'égalité entre traitements masculins et féminins a été accélérée par la « grève du baccalauréat » de 1927; cf. Alain Dalançon, Histoire du SNES, T. 1: Plus d'un siècle de mûrissement, des années 1840 à 1966/67, Paris, IRHSES, p. 42. 
De plus, le service des agrégées n'est pas spécialisé sur une chaire ou une discipline, comme celui des agrégés, ce qui accroît la lourdeur des préparations d'une année sur l'autre.

Après l'obtention de l'égalité des traitements, la Société des agrégées est persuadée d'obtenir rapidement l'égalité des services. Mais la crise économique retarde le processus, en dépit de l'accord de la rue de Grenelle. La Société dénonce la « dictature des Finances » (1). L'assemblée générale du 29 mars 1931 décide donc de radicaliser l'action: elle vote l'abstention lors des fêtes de célébration du Cinquantenaire de l'enseignement secondaire féminin. Ce vote n'a pas lieu sans débat. La présidente Gabrielle Véroux est réticente, car ce mode de pression risque de mettre en difficulté les hauts fonctionnaires de l'Instruction publique, qui sont d'accord sur la question des maxima, tandis que les représentants du Budget en profiteront pour dire que les agrégées manquent singulièrement d'élégance. Catherine Schulhof insiste et emporte la décision. Ce vote montre l'ampleur de l'exaspération des agrégées. Il témoigne aussi de l'arrivée à la tête de la Société d'une nouvelle génération, moins timorée et plus engagée.

Le ministère ne peut dignement célébrer le Cinquantenaire en l'absence des représentantes des agrégées, et même de tous les autres professeurs, car $\mathrm{M}^{\text {Hle }}$ Bréchot, professeur au Havre et présidente de la Société des professeurs titulaires licenciés et certifiés, s'est dite prête à faire bloc et à ne pas participer. Les certifiées n'avaient pourtant rien à y gagner, au contraire, puisque la diminution du service des agrégées créerait une différence avec les certifiées, dont le maximum était jusqu'alors identique. L'esprit de solidarité des professeurs femmes n'a pas disparu. Le ministère préfère céder.

\section{La représentation au CSIP}

La composition du CSIP n'ayant pas été modifiée par la loi Camille Sée, l'enseignement secondaire féminin n'y est pas représenté. Même les antiféministes comme Jean Maillan sont pourtant favorables à l'accès des femmes aux droits moraux. Mais la Société des agrégées a privilégié les droits matériels. Il est vrai que la ques-

(1) Les Agrégées, n 32, octobre 1930, p. 4 et n³3, décembre 1930-janvier 1931, p. 4. 
tion de la représentation des professeurs de l'enseignement secondaire féminin au CSIP ne concerne pas seulement les agrégées.

C'est néanmoins la Société des agrégées qui organise des élections quand le ministère accepte une représentation officieuse des professeurs femmes, en 1921. Ces élections mettent en jeu toutes les catégories de professeurs de l'enseignement secondaire féminin, mais toutes les élues sont des agrégées. Aussi bien, quand les élus des agrégés au CSIP refusent de discuter de la réforme de l'enseignement secondaire féminin en l'absence d'une délégation féminine, en 1924, le ministère fait venir non pas les déléguées officieuses, mais une délégation de la Société des agrégées, ce qui est une forme de consécration. De même, quand Édouard Herriot nomme la vice-présidente de la Société, Gabrielle Véroux, à la section permanente du CSIP, en 1928. C'est finalement en 1933, à l'occasion d'une réforme du Conseil supérieur, qu'est acquis le principe d'une représentation de l'enseignement secondaire féminin. À cette date, la Société des agrégées a rempli l'essentiel de son programme. Cela contribue à modifier son attitude: elle continue à associer valorisation des agrégées et promotion de l'enseignement secondaire féminin, mais se place désormais dans une attitude moins offensive (conquérir les avantages des agrégés) que défensive (maintenir la distance avec les non agrégées). Comme à la Société masculine, se fait jour l'idée que le nivellement des catégories ne favoriserait pas le prestige de l'enseignement secondaire.

\section{UNE ÉVOLUTION AMBIGUE DANS LES ANNÉES 1930}

Parmi les dirigeantes de la Société, la génération des années 1920 avait passé des examens relevant de l'enseignement primaire. Deux présidentes, Élisabeth Butiaux et Gabrielle Véroux, avaient même débuté leur carrière dans l'enseignement primaire. De là, sans doute, un plus grand souci de ne pas couper la Société des agrégées des autres catégories d'enseignantes et un attachement plus grand à la pédagogie. À l'instar de sa présidente, Catherine Schulhof, la génération qui domine le bureau de la Société dans les années 1930 n'a connu que l'enseignement secondaire, et elle revendique plus nettement la primauté des agrégées. 
La première moitié des années 1930, du fait des restrictions budgétaires, est en outre propice aux rivalités entre les différentes catégories. Plus conservatrice du point de vue catégoriel, la Société cherche à rester progressiste du point de vue pédagogique et adopte, par ailleurs, une posture de gauche. La base est cependant réticente.

\section{Une orientation plus catégorielle mal assumée}

Les grandes conquêtes de la Société ont été menées en collaboration avec les certifiées. En dépit des tentatives de division, le personnel féminin a su faire bloc. Dans les années 1930, se dessine cependant une division des catégories. La personnalité de la présidente de la Société des agrégées, Catherine Schulhof, qui a toujours été très fière de son agrégation, n'est pas étrangère à cette orientation.

Le résultat est un retournement par rapport à la situation primitive: en 1905, les femmes étaient restées unies; dans les années 1930, la Société des agrégées se rapproche de son homologue masculine, tandis que la Société des certifiées s'est alliée aux chargés de cours masculins pour former la Société des professeurs titulaires licenciés et certifiés des enseignements secondaires féminin et masculin. Le titre prime. L'aboutissement de ce processus est l'élection, en 1938, de Geneviève Bianquis, professeur à la faculté des lettres de Dijon, au comité de la Société des agrégées de l'enseignement secondaire: comme à la Société des agrégés, la qualité d'agrégée finit par l'emporter sur la fonction dans l'enseignement secondaire.

Une des premières manifestations du plus grand esprit catégoriel de la Société se situe en 1928, quand celle-ci rappelle au ministère la règle de la priorité des agrégées dans la nomination des directrices. Cet état d'esprit s'accentue dans les années 1930. En 1932, la Société suit son homologue masculine dans la revendication de priorité des agrégés pour l'obtention d'une chaire en lycée. Ce vœu n'est pas platonique: en octobre 1934, Catherine Schulhof obtient du directeur de l'Enseignement secondaire, Francisque Vial, une rectification du mouvement au profit d'une agrégée qui demandait un poste depuis cinq ans et qui avait vu nommer une licenciée. Détail significatif: la démarche a été faite de concert avec le président de la Société des agrégés, Pierre Couissin. 
Même orientation concernant les chaires de philosophie. Il n'existe pas d'agrégation féminine de philosophie, puisque la dernière classe des études secondaires féminines ne comportait pas, à l'origine, l'étude de cette discipline. Après la guerre, de plus en plus de jeunes filles s'étant mises à préparer le baccalauréat, les établissements féminins sont amenés à faire appel à des agrégés de philosophie. La présence d'hommes dans les établissements de jeunes filles, cependant, n'est pas souhaitée. C'est pourquoi la circulaire du 8 mars 1920 nuance la dépêche ministérielle du 8 mars 1919, qui avait interdit les agrégations masculines aux jeunes filles, et les autorise à passer les agrégations de philosophie et de grammaire. En 1921, devant la ferme opposition de la Société des agrégées, le ministre renonce à créer une agrégation féminine de philosophie (1). En 1924, par arrêté du 17 mars, il se résout à autoriser les jeunes filles à passer toutes les agrégations masculines (2). Cependant, en attendant que soit constitué un vivier suffisant d'agrégées de philosophie, le ministère continue d'user de divers expédients pour remplir les chaires: il fait appel à des agrégés masculins et surtout à des agrégées féminines de lettres. Mais à partir des années 1930, les pouvoirs publics cherchent à nommer des femmes philosophes. Les agrégées de philosophie restant en trop petit nombre, il est fait appel à des licenciées de philosophie. Résultat: des agrégées (de lettres) sont remplacées par des licenciées (de philosophie). Reçue par le directeur de l'Enseignement secondaire, le 8 décembre 1932, la Société des agrégées proteste. Il est remarquable que la défense catégorielle des agrégées féminines l'ait conduite, pour le coup, à minorer l'importance de la spécialisation.

En mai 1939, la Société dénonce la nomination de déléguées licenciées dans des lycées parisiens, au motif que des agrégées acceptent de faire des heures supplémentaires. Cette critique peut étonner, puisque la Société s'était prononcée pour la transformation d'heures supplémentaires en création de postes. Il est vrai que la Société refuse que ces groupements d'heures aboutissent à la « création de postes au rabais ».

(1) Les Agrégées, $\mathrm{n}^{\circ}$ 2, janvier 1921, p. 18 et n 3, avril 1921, pp. 5-6, 8 et 12.

(2) Françoise Mayeur, op. cit., p. 412, et Loukia Efthymiou, « Le genre des concours », Clio. Histoire, femmes et sociétés, $\mathrm{n}^{\circ} 18$, Coéducation et mixité, 2003, pp. 101-103. 
Le nouvel état d'esprit de la Société des agrégées aurait pu la rapprocher de son homologue masculine. En fait, le rapprochement est forcément limité, tant les considérations misogynes restent présentes dans la Société masculine, comme l'illustre le rapport publié, le 15 mars 1936, par son bulletin L'Agrégation. Sous le titre de « Sabotage de l'enseignement secondaire », ce rapport rédigé par le président de la Société, Pierre Couissin, condamne les mesures d'économie du ministère, mais contient également des remarques hostiles à la présence des femmes dans les lycées de garçons, comme si cette présence contribuait, elle aussi, à l'abaissement de l'enseignement masculin. La Société des agrégées proteste:

[L'assemblée générale] constate avec surprise que ce rapport ne signale, à la même rubrique, ni la présence des professeurs hommes dans l'enseignement féminin, ni la suppression de nombreuses classes dans les établissements féminins, qui entraine la constitution de nombreuses classes mixtes dans les établissements masculins (1).

Il arrive en outre à la Société des agrégées de se montrer plus conciliante, sur le plan catégoriel, que son homologue masculine, comme en témoigne son acceptation en 1935, après bien des hésitations, de l'idée d'instituer un cadre supérieur pour les non agrégés (2). Marthe Fauré, qui a promu cette idée, n'est toutefois pas réélue en 1938 aux élections au CSIP, et son militantisme en faveur de la FGE (CGT) n'est certainement pas seul en cause: la majorité des agrégées se montre hostile aux mesures qui pourraient menacer la suprématie de leur titre (3).

(1) Les Agrégées, $\mathrm{n}^{\circ}$ 54, avril-mai 1936, p. 18.

(2) Les chargés de cours de lycée, licenciés dans les établissements masculins, licenciées et certifiées dans les établissements féminins, ne peuvent espérer être promus dans le cadre de Paris, qui a été réservé aux agrégés aux termes du décret du 24 juillet 1913, sur lequel ni la Société des agrégés ni son homologue féminine n'acceptent de revenir.

(3) Ce sont les agrégées des départements qui peuvent se sentir les plus atteintes par un cadre supérieur qui donnerait aux non agrégés un traitement similaire au leur ou les en rapprocherait. Si cette majorité ne s'est pas manifestée lors du vote de l'assemblée générale de la Société, c'est que les provinciales sont rares à s'y déplacer. Le bureau de la Société le déplore régulièrement, mais ne semble pas vouloir réellement remettre en cause la prééminence des Parisiennes: lors de l'AG de 1933, on s'émeut de constater que les provinciales ne sont que quatre, mais on n'envisage pas pour autant le remboursement des frais de transport, même aux déléguées de section, sous prétexte que les finances de la Société ne le permettent pas et qu'une revalorisation de la cotisation est exclue, pour ne pas nuire au recrutement. Le comité lui-même est statutairement composé de deux tiers de Parisiennes et d'un tiers de provinciales, proportion inverse des effectifs (et de celle qui est de règle au comité de la Société masculine). 
C'est pourquoi la Société des agrégées, comme son homologue masculine, ne se montre pas favorable à l'idée de créer un concours de recrutement autre que l'agrégation à destination des licenciés. De même, elle se défie des propositions de réforme de ce concours, qui pourraient remettre en cause l'assimilation entre hautes compétences scientifiques et aptitude pédagogique sur laquelle les agrégés fondent leur prééminence. Elle finit donc par se ranger, dans les années 1930, à la position de la Société masculine. Elle avait certes accepté en 1926, à l'inverse de cette dernière, le projet des Compagnons de l'Université nouvelle de créer un certificat comportant des épreuves pédagogiques et professionnelles (sous la forme d'un stage), pourvu que ce certificat prît la forme d'un concours. En 1930, cependant, quand elle répond au questionnaire sur la préparation à l'agrégation que lui adresse son homologue masculine, elle exprime son opposition à une accentuation de la préparation pédagogique et préconise le maintien de la préparation existante (qui se résume au stage d'agrégation). Elle ne veut pas entendre parler de conférences pédagogiques. Selon elle, par ailleurs, il n'y a pas lieu que la préparation pédagogique commence dès la licence. Dans l'élan du Front populaire, néanmoins, Marthe Fauré fait accepter le principe d'une « mise à l'étude d'une réforme des concours d'agrégation et des conditions de recrutement homogènes de tous les maîtres du second degré » (1). Le projet est en lien avec la réforme Jean Zay. À la rentrée d'octobre, toutefois, il n'est plus question que d'un examen de classement des licenciés, dont les modalités ne sont pas précisées. Et en 1938, la Société des agrégées se borne à demander des facilités de préparation à l'agrégation pour les jeunes maîtres d'internat, les répétiteurs et les professeurs de collège. La Société des agrégés ne dit pas autre chose. Ces hésitations de la Société féminine illustrent la contradiction entre la montée du sentiment catégoriel et la volonté de continuer à être une association réformiste allant dans le sens du progressisme.

\section{Progressisme idéologique}

C'est, paradoxalement, au moment où se dessine une orientation plus catégorielle et où la solidarité féminine devient moins évidente que la Société des agrégées se rapproche d'organisations féministes, dans leur version réformiste. Dans les années 1920, le bulletin se

(1) Les Agrégées, n 59, mai-juin 1937, p. 19. 
bornait à rapporter, dans sa partie non officielle, les actions que ses dirigeantes avaient menées, à titre individuel, au sein d'organisations féministes. En 1929, un pas est franchi : l'adhésion au Conseil national des femmes (CNF) est cette fois adoptée par l'assemblée générale, à l'unanimité (1). Ce revirement n'est probablement pas le résultat du dynamisme des associations féministes réformistes, qui tendent au contraire à se scléroser (2). Il peut davantage s'expliquer par l'arrivée à la tête du bureau de la Société d'une équipe plus engagée et plus marquée à gauche, en particulier du point de vue syndical. Par ailleurs, le fait que les grandes revendications des années 1920 aient été satisfaites peut conduire la Société à élargir son champ d'action.

Comme le CNF est lui-même une section du Conseil international des femmes, qui regroupe 300 sociétés féminines, la Société est amenée à participer à l'organisation du Congrès international des activités féminines qui se tient à Paris en 1937, à l'occasion de l'Exposition universelle. L'année suivante, la présidente de la Société, Catherine Schulhof, collabore avec le CNF pour une action en faveur de l'émancipation des femmes d'Afrique du Nord par l'enseignement. De même, à l'appel du CNF, Catherine Schulhof agit en faveur de la reconnaissance de la capacité civile des femmes mariées, action qui contribue au vote de la loi René Renoult (3).

On est donc surpris de constater que, lors de l'assemblée générale de 1938, la Société refuse de s'occuper de la question des congés de maternité au motif que ce serait un problème d'ordre général, dépassant la sphère d'activité corporative (4). On a l'impression que ces femmes éprises d'émancipation, de surcroît le plus souvent célibataires, considèrent avec peu d'aménité un droit qui rappellerait la vocation procréatrice de la femme, alors que la concurrence féminine sur le marché du travail est dénoncée et que les organisations sociales catholiques prônent le « retour» des femmes au foyer (5). De ce point de

(1) Les Agrégées, nº 26, mars 1929, p. 5.

(2) Christine Bard, Les filles de Marianne. Histoire des féminismes, 1914-1940, Paris, Fayard, 1995, p. 383.

(3) La loi Renoult du 18 février 1938 étend les droits civils de la femme mariée. Le mari reste le chef de famille et peut encore interdire à son épouse l'exercice d'un métier, mais la femme mariée a notamment le droit de faire des études et de passer un examen.

(4) Les Agrégées, $\mathrm{n}^{\circ}$ 62, mars-avril 1938, p. 145.

(5) Christine Bard, Les femmes dans la société française au XXe siècle, Paris, A. Colin, 2001, p. 68. 
vue, la réserve de la Société se trouve en décalage avec la position de la majorité du mouvement féministe pendant les années 1930 (y compris avec celle du CNF, auquel elle a adhéré), qui, certes, défend le travail féminin, mais cherche aussi à concilier maternité et travail (1).

Si la Société ne s'engage pas, en tant que telle, dans le combat pour le suffrage féminin, son action ne s'en inscrit pas moins dans le courant des luttes que mènent les femmes progressistes. En juin 1936, la Société salue la nomination de femmes comme ministres. Elle se fait recevoir par $\mathrm{M}^{\text {me }}$ Brunschwicg, sous-secrétaire d'État à l'Éducation nationale, puis par $\mathrm{M}^{\mathrm{me}}$ Joliot-Curie, sous-secrétaire d'État à la Recherche scientifique, avant de rencontrer le ministre de l'Éducation nationale Jean Zay - avec qui elle entretiendra les meilleures relations. Cet hommage et cette précipitation font partie de son soutien discret au Front populaire. Il est vrai que les principales figures du bureau de la Société sont alors membres de la Fédération générale de l'enseignement (CGT).

L'engagement syndical des principales personnalités de la Société des agrégées lui donne, dans les années 1930, une coloration nettement progressiste. De là, lors de l'assemblée générale de 1933, une dénonciation de l'arrivée au pouvoir d'Hitler (ainsi que des lois xénophobes votées en France depuis 1932), à laquelle a tenu la cégétiste Marthe Fauré :

En présence des événements actuels, l'Assemblée générale de la Société des agrégées tient à déclarer qu'elle considère l'égalité de tous les nationaux d'un pays devant les lois comme un des principes indispensables de la civilisation et s'élève en particulier contre l'inégalité des droits en matière d'études, d'accession aux diverses professions ou aux fonctions publiques, d'après les différences de race, de religion ou d'opinion politique et sociale (2).

Cette motion est à mettre en relation non seulement avec l'importance des « cégétistes » au sein du bureau de la Société, mais aussi avec son engagement plus explicitement féministe. En septembre 1933, lors de son congrès à Chicago, le Conseil international des femmes, qui se veut pourtant apolitique (3) - comme la Société

(1) Cf. Anne Cova, Maternité et droits des femmes en France, XIXe-XXe siècles, Paris, Anthropos, 1997, p. 331.

(2) Les Agrégées, $n^{\circ}$ 43, juin 1933, p. 35.

(3) Catherine Jacques, « Construire un réseau international: l'exemple du Conseil international des femmes (CIF) », in Éliane Gubin et al. (dir.), Le siècle des féminismes, Paris, Éditions de l'Atelier/Éditions ouvrières, 2004, p. 131. 
des agrégées elle-même -, vote une motion du même type, qui affirme le droit de toute personne de tout sexe, de toute race et de toute nationalité à la sécurité de la vie, du travail, de la santé et de l'éducation (1).

Dominée par des femmes de gauche, la Société dénonce dans les années 1930 la politique de déflation. Comme au Syndicat national (mais non pas comme à la Société masculine), le discours se politise (2). La Société des agrégées inscrit cependant le progressisme de son discours dans la défense du travail féminin, à l'image d'organisations comme la Ligue des droits de la femme (3), à laquelle appartient Marthe Fauré. En 1935, la Société envoie une motion en faveur de la défense du travail féminin à Genève, au Bureau international du travail (BIT) et à l'Institut de coopération intellectuelle. Marthe Fauré prend même l'initiative de l'envoyer au secrétaire général de la CGT, Léon Jouhaux. Son influence est d'ailleurs marquée par le fait que le Bureau lui confie le soin, en octobre 1935, de rédiger un article, qui sert d'éditorial au bulletin de la Société, sur «Le droit des femmes au travail ». Marthe Fauré conclut en indiquant « à nos sociétaires la position d'un certain nombre d'associations corporatives et professionnelles sur une question qui a fait l'objet d'un des vœux les plus importants de notre Assemblée générale » (4). Suit l'explication de la position du S3, de la Confédération des travailleurs intellectuels (CTI), de la CGT, du BIT, positions favorables au travail des femmes, puis de la position de syndicats chrétiens favorables au principe de la femme au foyer.

Nous conseillons à nos collègues, conclut Marthe Fauré, de s'informer, avant d'adhérer à un groupement corporatif ou professionnel, d'ordre social ou même d'ordre politique ou idéologique, de la position de ce groupement sur la question du droit au travail des femmes, afin d'adhérer en connaissance de cause (5).

L'engagement de la Société devient donc plus apparent. Soucieuse du rappel du droit au travail (et pas seulement pour les femmes), la Société des agrégées accueille avec une faveur à peine dissimulée la victoire du Front populaire. En 1937, elle approuve d'ailleurs nette-

(1) Christine Bard, Les filles de Marianne..., op. cit., p. 292.

(2) Paul Gerbod, «Associations et syndicalismes universitaires de l'enseignement secondaire public, 1928-1937 », Le mouvement social, octobre-décembre 1970, pp. 79-110.

(3) Christine Bard, Les filles de Marianne..., op. cit., p. 317.

(4) Les Agrégées, $\mathrm{n}^{\circ}$ 52, octobre-novembre 1935, p. 5.

(5) Ibid., p. 7. 
ment le projet de réforme de Jean Zay. Certes, à cette date, la Société masculine aussi accepte globalement le projet. Mais l'approbation de la Société féminine est plus profonde, puisqu'elle admet des classes d'orientation communes ayant des maittres volontaires provenant des divers ordres d'enseignement. Elle souscrit à l'idée d'orientation, que méconnaît au contraire son homologue masculine (1).

La Société des agrégées n'oublie d'ailleurs pas la nécessité d'une réorganisation générale de l'enseignement, afin d'assurer le passage du premier au second degré, de permettre une judicieuse orientation des élèves, de mettre fin à l'incohérence qui trouble les enseignements du second degré, de préparer l'entrée des élèves du second dans le troisième degré (2).

Contrairement à son homologue masculine, la Société considère avec intérêt, par ailleurs, les propositions d'utilisation des méthodes audiovisuelles: signe d'une persistance du progressisme pédagogique de l'enseignement secondaire féminin? Marthe Fauré, qui s'était distinguée en faveur de la classe d'orientation, n'est toutefois pas réélue au CSIP. On peut se demander s'il n'y a pas un décalage entre le progressisme du bureau et les sentiments de la base. Au sein même du bureau, les motions idéologiques sur la défense du travail féminin peuvent, au reste, servir d'alibi idéologique à l'orientation plus conservatrice de la Société du point de vue catégoriel.

\section{LA FUSION AVEC LA SOCIÉTÉ MASCULINE}

Rien ne permettait pourtant de prévoir, avant-guerre, la fusion des deux associations. En 1937, dans son rapport moral, Catherine Schulhof indiquait que les relations avec la Société masculine étaient « nulles».

Comme son homologue masculine, la Société des agrégées cesse son activité en juin 1940. En 1941, sa présidente, Catherine Schulhof, est écartée de l'enseignement en raison de ses origines juives. La Société reprend vie le 16 juillet 1945, avec la tenue d'une assemblée générale. En apparence, tout se passe comme s'il n'y avait eu aucune rupture. Contrairement à celui de son homologue masculine, le bureau de la Société est pratiquement inchangé. Avec les syndicats, et en particulier avec le Syndicat national de l'enseignement secondaire (SNES), membre de la Fédération de l'Éducation nationale (FEN), la

(1) Les Agrégées, n 59, mai-juin 1937, p. 17.

(2) Les Agrégées, $\mathrm{n}^{\circ}$ 56, octobre-novembre 1936, p. 7. 
Société établit des relations régulières, qui témoignent de son influence: $M^{\text {elles }}$ Lac et Schulhof, respectivement secrétaire générale et présidente de la Société, sont membres de la Commission corporative du SNES, lequel est représenté officiellement à la Société par $\mathrm{M}^{\mathrm{lle}}$ Fauré. Autre manifestation de l'audience retrouvée: toutes les agrégées élues au Conseil supérieur de l'Éducation nationale appartiennent à la Société. La tradition d'ouverture pédagogique est par ailleurs maintenue, comme en témoigne l'intérêt pour l'expérience des classes nouvelles, auxquelles la Société masculine est pour sa part hostile (1). Pourtant, $\mathrm{M}^{\mathrm{lle}} \mathrm{Lac}$, qui est à bien des égards la représentante patentée de la Franco-ancienne (2) à la Société des agrégées, n'a pas eu de peine à montrer que la non-spécialisation des services qu'implique la bivalence est contraire à tout l'effort qu'a fait le mouvement corporatiste féminin depuis ses origines. L'acceptation de la bivalence par la Société des agrégées n'en est que plus remarquable. Cependant, dès qu'il s'agit de défendre les chaires, la Société se montre intransigeante, ce qui la conduit à condamner le projet Langevin-Wallon:

Alarmée par des projets qui tendent, semble-t-il, à écarter les agrégés de tout ou partie de l'enseignement secondaire [...], l'Assemblée générale ne saurait admettre que l'agrégation ne fût pas le titre par excellence du professeur de l'enseignement secondaire, de la $6^{\mathrm{e}}$ à la terminale (3).

À vrai dire, l'esprit catégoriel est devenu plus patent. Ainsi la Société estime-t-elle qu'il faut augmenter le nombre de places à l'agrégation, car « un grand nombre de chaires de l'enseignement secondaire sont tenues par des déléguées qui donnent peu de garanties de savoir et de compétence » (4). Dans ces conditions, la Société adopte souvent le point de vue de son homologue masculine. L'observateur que délègue celle-ci acquiert de plus en plus d'influence. Lors de la séance du bureau du 10 janvier 1946, M $^{\text {lle }}$ Fauré, membre de la Fédération générale de l'enseignement (FGE), défend le projet sur les traitements de son syndicat et paraît convaincre ses collègues.

(1) Les Agrégées, $\mathrm{n}^{\circ} 76,2^{\mathrm{e}}$ trimestre 1947, p. 18. Promues par le directeur de l'Enseignement secondaire, Gustave Monod, les classes nouvelles prévoient la pédagogie active et la bivalence (pour mieux suivre et connaître les élèves) et instituent des programmes tournés vers l'étude du « milieu naturel et humain ».

(2) Association de spécialistes réunissant des professeurs de français et de langues anciennes.

(3) Les Agrégées, $\mathrm{n}^{\circ}$ 71, 1er trimestre 1946, p. 17.

(4) Ibid. En dépit de son progressisme pédagogique, la Société des agrégées se rapproche de celle des agrégés par l'assimilation entre savoir et compétence. 
Jacquemart, vice-président de la Société masculine, parvient à renverser l'opinion du bureau de la Société féminine et à faire refuser le projet de revalorisation de la FGE, qui établit une échelle des traitements de seulement 1 à 2 entre les instituteurs et les agrégés (1).

Dans ces conditions, le processus de fusion devient d'actualité. L'anémie de la vie de la Société, qui n'est certes pas nouvelle mais a pris une dimension préoccupante (il y a plus de places aux élections au comité que de candidates), s'explique par le fait que la base ne croit plus en la nécessité d'une société féminine séparée. Dès 1945, des sections ont fusionné. Estimant que la Société féminine a des préoccupations qui lui sont propres, Catherine Schulhof se montre réticente envers l'idée d'une fusion, qui risque de surcroît d'être une absorption, les agrégés étant deux fois plus nombreux que les agrégées (3000 adhérents à la société masculine contre 1500 à la société féminine). Voulue par la base, la dynamique de la fusion est cependant irréversible. Il est frappant que ce soit la Société masculine qui soit la plus déterminée en ce sens, quand on se souvient de son refus d'intégrer avant-guerre même les « agrégées masculines ». Le bureau de la Société féminine finit par consentir à la fusion, après la rédaction d'un rapport commun (2): la Société unifiée comprendra une Section des intérêts féminins, composée aux deux tiers de femmes. Cette section ne sera pas un État dans l'État, dans la mesure où c'est le comité de la Société unifiée, composé pour deux tiers d'hommes, qui tranchera en dernier ressort. En 1948, les deux sociétés adoptent ces propositions et fusionnent.

C'est tout naturellement Catherine Schulhof qui est appelée à la présidence de la Section des intérêts féminins (SIF), dont la grande affaire va être celle des géminations de classes terminales, qui s'opèrent toujours au profit des lycées masculins (3). Alors que l'enseignement secondaire féminin a vaincu tous les préjugés contre l'éducation des filles par l'État, voici qu'il serait menacé non plus par la méfiance des familles, mais par la politique de ce même État.

(1) Sur le « projet Collignon » de revalorisation des traitements proposé au nom de la FGE, $c f$. Guy Brucy, Histoire de la FEN, Paris, Belin, 2003, pp. 85-87.

(2) Raphaël Barquissau et Alberte Mélot, L’Agrégation, n 293, mars 1948, pp. 150-151.

(3) Faute d'effectifs suffisants ou pour des raisons d'économies, les pouvoirs publics sont parfois conduits à fermer les classes terminales des établissements féminins et à autoriser les jeunes filles à se rendre dans l'établissement masculin le plus proche. 
N'aboutit-on pas, en outre, à instaurer, de façon larvée, une mixité dont personne n'a discuté au préalable? Par ailleurs, n'est-il pas injuste et contraire à l'intérêt de l'enseignement que, faute parfois d'agrégés, des classes terminales soient confiées à des licenciés plutôt qu'à des agrégées, fait habilement remarquer la présidente de la SIF ? Il est probable que certains hommes, en sous-main, persistent à penser que les agrégées sont d'un niveau inférieur; mais le comité de la Société soutient officiellement les revendications féminines. Quand Catherine Schulhof, excédée de voir les lycées féminins « découronnés », se fait recevoir par le ministre sans se faire accompagner par le président de la Société, les protestations sont cependant vives. Amère, Catherine Schulhof démissionne. Elle est remplacée par Marguerite Schwab-Sommer, qui, très rapidement, pose la question du maintien de la SIF. Un an après, en 1956, celle-ci se dissout.

La disparition de la SIF est à mettre en relation avec le reflux des revendications féministes dans les années 1950. Sylvie Chaperon évoque une époque de " désillusion », marquée par la stagnation des effectifs des groupements féministes (1). La dissolution de la SIF peut toutefois être interprétée aussi comme une étape dans le processus d'assimilation des hommes et des femmes. De toute façon, l'explosion scolaire ne nécessite plus que chaque enseignement, masculin et féminin, protège ses chaires. C'est donc surtout le contexte, scolaire et catégoriel, qui s'est modifié.

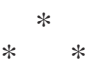

Comme son homologue masculine, la Société des agrégées s'est d'abord fondée pour peser sur la Fédération nationale des professeurs de lycée. Pour y obtenir la majorité, elle a dû s'unir avec le personnel féminin. Au départ, en effet, l'adversaire, ce n'était pas le non agrégé, comme c'était le cas du côté de la Société masculine, mais l'agrégé masculin, qui déniait aux titres féminins une valeur égale à celle des siens. Au cours de cette première phase, certifiées et agrégées se sont épaulées mutuellement: avec le soutien des agrégées, les certifiées ont obtenu l'assimilation aux licenciés en 1921; dix ans plus tard, c'est avec le soutien des certifiées, qui n'avaient pourtant rien à y gagner, que les agrégées ont conquis l'égalité des maxima de service avec les agrégés.

(1) Sylvie Chaperon, Les années Beauvoir, 1945-1970, Paris, Fayard, 2000, p. 211. 
À cette date, toutefois, une recomposition s'opère. Le personnel féminin n'apparaît plus aussi uni. Les certifiées forment une association avec leurs collègues masculins non agrégés. De son côté, la Société des agrégés demande à son homologue féminine sa collaboration pour lutter contre la revendication des chargés de cours d'un accès au cadre de Paris. Lors des congrès du Syndicat national, une alliance électorale s'établit désormais entre les deux sociétés des agrégés, qui leur permet d'imposer leurs vues. La fusion des deux sociétés est cependant inconcevable, l'alliance ponctuelle n'empêchant pas la persistance des préjugés masculins. Par ailleurs, la Société féminine assume mal sa dérive catégorielle, et finit par accepter, contrairement à son homologue masculine, le principe d'un cadre supérieur des non agrégés. En 1936, dans l'élan du Front populaire, la Société des agrégées renoue avec la générosité en soutenant la réforme du cadre des répétitrices-préparatrices. Il semble néanmoins que la base soit plus réticente que la direction à accorder des avantages aux professeurs non agrégés. On peut même se demander si la phraséologie progressiste de la direction de la Société n'a pas aussi pour vertu de masquer l'orientation catégorielle.

Au total, quels sont les points communs et les différences avec la Société masculine ? De même que cette dernière, la Société des agrégées s'est fondée pour la défense d'intérêts matériels. Comme elle, elle se prétend responsable de l'enseignement secondaire, féminin en l'occurrence. Mais justement: la Société masculine s'estime responsable de la valeur de l'enseignement secondaire dans son ensemble; sa hantise, c'est la primarisation. La lutte pour le prestige de l'agrégation se justifie par le maintien de la spécificité de l'enseignement secondaire. La Société des agrégés se fonde donc contre les catégories inférieures, en réaction aux prétentions des non agrégés et, in fine, des primaires. De là une tonalité conservatrice - au reste mal assumée par sa majorité de gauche. La Société féminine se fonde pour obtenir l'égalité entre hommes et femmes. De là une tonalité progressiste (même si l'orientation plus à gauche de sa direction se conjugue avec une vie interne moins démocratique). Alors que la Société masculine déplore toujours la fin de l'âge d'or, la Société féminine regarde vers l'avenir. Sa finalité est certes plus catégorielle que celle de son homologue masculine. Le titre de leurs publications respectives en témoigne: Les Agrégées soutiennent explicitement un personnel, L'Agrégation prétend défendre un principe. Et pourtant, la Société féminine est plus intéressée par les questions pédagogiques 
que son homologue masculine. Dans les années 1920, deux de ses présidentes suivent les travaux de la Société française de pédagogie. De là, sans doute, une plus grande ouverture aux réformes de l'enseignement, réforme Jean Zay sous le Front populaire, puis classes nouvelles à la Libération. Cependant, ce réformisme est peut-être davantage celui des dirigeantes, proches de la FGE, que celui de la base. Par ailleurs, il s'arrête dès que les chaires sont menacées, comme le montre l'opposition au projet Langevin-Wallon. Sur ce point, la Société des agrégées rejoint son homologue masculine, attachée à un enseignement long d'imprégnation lente. Dans ces conditions, l'essentiel du programme de la Société féminine ayant été réalisé, la fusion des deux sociétés est logique, dans la mesure où la question essentielle, après la Libération, est celle de la réforme de l'enseignement, qui porte en elle la question de la place de l'agrégé. Dans la Société unifiée, en 1948, l'influence de Marthe Fauré, qui avait œuvré pour l'acceptation des classes nouvelles, cède la place à celle de $\mathrm{M}^{\mathrm{lle}} \mathrm{Lac}$, qui avait refusé les classes nouvelles au nom de la spécialisation des chaires et va contribuer, grâce à son ascendant sur le président Pierre Bennezon, à radicaliser le discours de la Société sur la place des agrégés et la réforme de l'enseignement. C'est le signe que pour les agrégées, du moins pour celles qui adhèrent à la Société des agrégés, l'enjeu principal est désormais celui de la défense de leurs privilèges et de la préservation de l'enseignement secondaire traditionnel, non celui de la perpétuation de la tradition progressiste de la Société des agrégées.

Cependant, cela ne signifie pas qu'elles se voient désormais uniquement comme des agrégées et non comme des femmes professeurs. En témoigne le fait que Geneviève Zehringer a été très fière, en tant que femme, d'avoir accédé à la présidence de la Société en 1990 et qu'elle a même proposé son soutien à la secrétaire générale du SNES, Monique Vuaillat, en butte, comme elle, à l'hostilité du ministre de l'Éducation nationale Claude Allègre: héritage de la solidarité féminine entre une agrégée et une non agrégée ? Monique Vuaillat, cependant, a refusé d'accepter un appui qu'elle a dû juger embarrassant. En l'occurrence, les divergences doctrinales ont été plus importantes que la solidarité féminine. 